\title{
Postmortem imaging in paediatric radiology - the French perspective
}

\author{
Marianne Alison
}

Received: 25 April 2014 / Accepted: 10 July 2014 / Published online: 23 August 2014

(C) Springer-Verlag Berlin Heidelberg 2014

Applying postmortem imaging in paediatric radiology is challenging. The wide spectrum of paediatric imaging includes both prenatal and postnatal settings (forensic or not), as well as different subspecialties such as neurological, cardiovascular, thoracoabdominal and musculoskeletal imaging. Most paediatric radiologists do not have all these skills and postmortem imaging requires a large team or network involving all these subspecialties. For example, experience in obstetric scanning and in vivo fetal MRI helps for fetal postmortem imaging analysis. Regular multidisciplinary team discussions with radiologists, pathologists and clinicians could help improve diagnosis. Postmortem imaging should be better taught and acquisition protocols should be standardized.

A further challenge is to differentiate normal postmortem imaging changes from pathology in such a wide setting. Larger studies should also be performed to compare postmor- tem imaging with conventional autopsy to improve paediatric radiologists' knowledge of normal postmortem changes. These studies appear necessary before postmortem imaging can be used on a wider scale or substitute for conventional autopsy in case of parental refusal.

However, several barriers limit the application of postmortem imaging in paediatric radiology in France: the lack of availability of MRI equipment, the need to perform these examinations outside normal working hours, the fact that these examinations are time-consuming for both acquisition (MRI) and for analysis (MRI and CT), and a shortage of paediatric radiologists. Moreover, most institutions do not recognize this activity and specific cost should be implemented for such examinations. Finally, postmortem imaging cannot substitute for conventional autopsy and its complete histological analysis, even if targeted biopsy could be performed.
M. Alison ( $\square)$

Service d'Imagerie Pédiatrique, Hôpital Robert Debré, AP-HP,

Université Paris Diderot - Sorbonne Paris Cité,

48 Bd Sérurier, 75019 Paris, France

e-mail: marianne.alison@rdb.aphp.fr 